Wilfrid Laurier University

Scholars Commons @ Laurier

\title{
The Role of Behavioral Dynamics in Determining the Patch Distributions of Interacting Species
}

\author{
Peter A. Abrams \\ University of Toronto \\ Ross Cressman \\ Wilfrid Laurier University, rcressman@wlu.ca \\ Vlastimil Křivan \\ Academy of Sciences of the the Czech Republic
}

Follow this and additional works at: https://scholars.wlu.ca/math_faculty

\section{Recommended Citation}

Abrams, Peter A.; Cressman, Ross; and Křivan, Vlastimil, "The Role of Behavioral Dynamics in Determining the Patch Distributions of Interacting Species" (2007). Mathematics Faculty Publications. 25.

https://scholars.wlu.ca/math_faculty/25

This Article is brought to you for free and open access by the Mathematics at Scholars Commons @ Laurier. It has been accepted for inclusion in Mathematics Faculty Publications by an authorized administrator of Scholars Commons@ Laurier. For more information, please contact scholarscommons@wlu.ca. 


\title{
The Role of Behavioral Dynamics in Determining the Patch Distributions of Interacting Species
}

\author{
Peter A. Abrams, ${ }^{1, \star}$ Ross Cressman, ${ }^{2, \dagger}$ and Vlastimil Křivan ${ }^{3, \ddagger}$
}

1. Department of Ecology and Evolutionary Biology, University of Toronto, Toronto, Ontario M5S 3G5, Canada; 2. Department of Mathematics, Wilfrid Laurier University, Waterloo, Ontario N2L 3C5, Canada;

3. Department of Theoretical Ecology, Institute of Entomology, Biology Centre, Academy of Sciences of the Czech Republic, Branišovská 31, 37005 České Budějovice, Czech Republic

Submitted July 19, 2006; Accepted November 16, 2006;

Electronically published January 31, 2007

AвSTRACT: The effect of the behavioral dynamics of movement on the population dynamics of interacting species in multipatch systems is studied. The behavioral dynamics of habitat choice used in a range of previous models are reviewed. There is very limited empirical evidence for distinguishing between these different models, but they differ in important ways, and many lack properties that would guarantee stability of an ideal free distribution in a single-species system. The importance of finding out more about movement dynamics in multispecies systems is shown by an analysis of the effect of movement rules on the dynamics of a particular two-species-two-patch model of competition, where the population dynamical equilibrium in the absence of movement is often not a behavioral equilibrium in the presence of adaptive movement. The population dynamics of this system are explored for several different movement rules and different parameter values, producing a variety of outcomes. Other systems of interacting species that may lack a dynamically stable distribution among patches are discussed, and it is argued that such systems are not rare. The sensitivity of community properties to individual movement behavior in this and earlier studies argues that there is a great need for empirical investigation to determine the applicability of different models of the behavioral dynamics of habitat selection.

Keywords: adaptive movement, competition, dispersal, ideal free distribution, patch choice, metacommunity.

\footnotetext{
* E-mail: abrams@zoo.utoronto.ca.

† E-mail: rcressma@wlu.ca.

₹ E-mail: krivan@entu.cas.cz.
}

Am. Nat. 2007. Vol. 169, pp. 505-518. (c) 2007 by The University of Chicago. 0003-0147/2007/16904-41971\$15.00. All rights reserved.
Knowing how animals should distribute themselves among different habitats is important for understanding observed spatial distributions. The question is of growing significance because biological communities are increasingly being fragmented into "metacommunities" in partially isolated habitat patches (e.g., Holyoak et al. 2005). The "ideal free distribution" (IFD), an idea introduced by Fretwell and Lucas (1970; see also Fretwell 1972), has played a key role in analyses of between-patch distribution under local density dependence. The IFD for a single species is usually defined as a distribution in which two criteria are satisfied: all occupied patches are characterized by equal fitness, and this is greater than the fitness an individual would achieve were it to move to any unoccupied patch. Fretwell and Lucas's (1970) definition used "ideal" to refer to the assumption that the animals in question had complete and accurate information on conditions in different habitats and "free" to refer to the lack of constraints on or costs to movement. These two conditions are not always needed to achieve an IFD (Cressman and Krrivan 2006). Unfortunately, most of the analyses of the IFD have assumed movement of only a single species and have ignored interspecific interactions. Furthermore, they have largely ignored the nature of behavioral movement rules that might lead to an IFD. Several articles have considered whether IFDs will be attained in particular multispecies models with specific assumptions about between-patch movement; these include Schwinning and Rosenzweig (1990), Abrams (1999, 2000), and Krrivan and Sirot (2002). These studies have come to differing conclusions about whether each species' eventual distribution among patches satisfies the traditional definition of an IFD. A wide range of other studies not explicitly concerned with IFDs have adopted a variety of different behavioral models of movement (see reviews by Briggs and Hoopes [2004] and Grimm and Railsback [2005]).

Two recent articles in this journal (Cressman et al. 2004, hereafter CKG, and Cressman and Křivan 2006, hereafter $\mathrm{CK})$ have begun to address some of the limitations of previous IFD theory. CKG extended the definition of an IFD to encompass two species ( 1 and 2 ) in the context of 
a two-habitat (A and B) model. The species were characterized by habitat distributions, denoted $\{p, q\}$, giving the fractions of populations 1 and 2, respectively, located in habitat A. Here we call a distribution yielding equal fitness across occupied habitats for each species at a particular pair of population densities a "candidate IFD" for those densities. CKG define a two-species IFD as a candidate IFD that is stable to spatial perturbations in the following sense: for any perturbed distribution $\left\{p^{\prime}, q^{\prime}\right\}$ characterized by the same population densities, the fitness of at least one of the species at that perturbed state is lower than the fitness of the candidate IFD at the perturbed state. This work did not specify the dynamics by which $p$ and $q$ changed, leaving it unclear whether all types of movement that increased individual fitness would bring a system to the IFD. CKG did note that, to persist, an IFD must also be an equilibrium for population dynamics. Later, CK showed that a single-species system having two (or more) patches and strictly negative effects of density in each patch would reach an IFD by several classes of movement rules and that this IFD was uninvadable; that is, it was an evolutionarily stable strategy. Two properties were sufficient to reach the single-species IFD: no movement to poorerquality patches and some movement to the best patch. However, CKG and CK still did not provide much guidance regarding the between-patch distributions expected in a generic metacommunity, in which movement behaviors of some or all species may not fall within the class that is guaranteed to reach a single-species IFD. Furthermore, these two works did not provide a description of the population dynamics that could result when a twospecies IFD (sensu CKG) did not exist.

This work shows that the habitat distribution that comes about in a metacommunity is often quite sensitive to the behavioral dynamics of movement. It shows that previous models of movement dynamics have differed in a number of important respects but that there is little empirical evidence that can be used to choose among these models when considering any particular system. It then uses an example of two competitors in a two-patch system (from CKG) to show that the differences between equally plausible movement models can lead to surprisingly large differences in population dynamics when two mobile species interact in a patchy environment. This article presents some general arguments why the phenomena exhibited in this example are unlikely to be rare. Finally, it draws conclusions about the general importance of incorporating adaptive behavior into community models and the importance of more empirical work to distinguish among the many potential models of habitat selection if we are to understand population dynamics within metacommunities.

\section{Previous Models of Habitat Selection Dynamics in Systems with Several Interacting Species}

Here we classify and analyze several models that have been used to describe an animal's choice of location when fitness differs between habitats. These models span the range of published models in terms of several important binary characteristics. Theoretical studies on habitat selection of interacting species that included explicit dynamics for habitat choice for at least one species include Bernstein et al. (1988, 1991, 1999), Schwinning and Rosenzweig (1990), Ives (1992), Abrams and Matsuda (1993, 2004), van Baalen and Sabelis (1993, 1999), Matsuda and Abrams (1994), Abrams (1999, 2000, 2006a, 2006b), Alonzo (2002), de Roos et al. (2002), Persson and de Roos (2003), and Armsworth and Roughgarden $(2005 a, 2005 b)$. Adaptive movement has also been incorporated into a large number of simulation models of animals in particular systems. Fryxell et al. $(2004,2005)$ is representative of a number of spatially explicit simulations of patch selection involving adaptive habitat choice that have compared model predictions to observations of spatial distributions. Many previous models of interacting species with flexible behaviors have ignored the dynamics of change in location and have simply assumed that those species instantaneously reach an IFD (Rosenzweig 1991; Křivan 1997; Křivan and Sirot 2002).

Most of the models of habitat choice mentioned in the preceding paragraph can be classified into several categories in regard to the following properties: (1) Is movement out of a patch a function of conditions in only the current patch or of conditions in other patches as well? (2) Is movement to a poorer-quality patch possible? (3) Is fixation of habitat location possible? (4) Is the timescale of between-patch movement rapid or slow relative to demographic processes? Different models from the literature cited in the preceding paragraph have differed in how they address these questions. Perhaps the one common feature of all models listed is that the instantaneous rate of movement (for continuous models) or the probability of movement (for discrete models) of an individual increases as its fitness in its current patch decreases, all else being equal. Because most of the models have sought to relate their conclusions to Fretwell and Lucas's (1970) work, the models typically have assumed that movement is effectively instantaneous and cost free.

The fourth question has defined the form of the model that defines movement in most previous studies of habitat choice. In "slow" models, the fitness of an individual is characterized by the conditions in its current patch. In "rapid" models, demographic processes are slow on the timescale of movement, and an individual's short-term fitness is characterized by the proportions of time spent in the different patches. It is clear that the frequency of 
movement is important in deciding which framework to use. Models that use lifetime fitness measures, like those of Alonzo (2002), are likely to be needed to distinguish which of these two idealized cases is more appropriate and to investigate intermediate cases. It is important to note that individuals may be slow in changing the proportional time allocation, even though they move between patches frequently (i.e., are described by a rapid movement model). Similarly, individuals may change location very rapidly when there is a fitness benefit to doing so under the slow model, but they should then stay in that patch long enough that their fitness while in the patch becomes mainly a function of conditions in that patch.

A relatively general form that encompasses several rapid models for the case of two habitats (A and B) describes the dynamics of the proportion of time spent in habitat A (denoted $p)$, using the equation

$$
\frac{d p}{d t}=v(p)\left(\left.\frac{d W\left(p^{\prime}, p\right)}{d p^{\prime}}\right|_{p^{\prime}=p}\right)+\mu(p) .
$$

Here $W$ is the per capita growth rate (i.e., fitness) of a "behaviorally deviant" individual with habitat distribution $p^{\prime}$ in a resident population characterized by $p$; the derivative is evaluated where $p^{\prime}=p$. The function $v(p)$ scales the rate at which the habitat distribution changes based on the current time allocation. In the absence of any nonadaptive habitat change $(\mu)$, the function $v(p)$ must be such that $p$ remains between 0 and 1 (Abrams 1999). The function $\mu$ represents change in distribution that is unrelated to fitness, including random movement. Without $\mu, p$ can never change in a direction that reduces fitness, but with a nonzero $\mu$ such change is possible. Because all habitats are assumed to be visited frequently in rapid models, the first of the four questions listed above is irrelevant; conditions in other patches generally affect movement out of a given patch. Abrams (2003, 2006a, 2006b) and Abrams and Matsuda (2004) use versions of equation (1) with a nonzero $\mu$. Models similar to equation (1) have more often been used to model the dynamics of behavioral traits within a habitat rather than between-habitat movements.

Slow models assume that an individual's fitness can be characterized by the patch it currently occupies rather than by a fraction of time spent in that patch. Slow models have been adopted in the majority of spatial simulation models of particular species (e.g., Mooij et al. 2002; Fryxell et al. 2004, 2005 and references therein). The movement rule that appears to be most common in the theoretical literature is one in which the movement rate out of a patch is purely a function of fitness of individuals within that patch. The number of individuals leaving patch $i$ is then described by $N_{i} f\left(W_{i}\right)$, where $N_{i}$ is the number of individuals in patch $i$ and $f$ is a nonnegative function that decreases with $W_{i}$ and either approaches or equals 0 when $W_{i}$ is sufficiently large. When only two patches are present, movement out of one is necessarily into the other. With three or more patches, movement into a patch from the pool of emigrants may be random or random within some subset of patches (e.g., those having higher than the mean fitness, as in Bernstein et al. 1999), or it may be an increasing function of the fitness in that patch relative to the mean of the other patches. If patch quality can be assessed remotely (the assumption in Fretwell and Lucas's [1970] original analysis), the per capita movement out of one patch and into another is an increasing function of the difference in fitness between the second patch and the first in a two-patch system (Abrams 2000). Abrams (2000) represented the net movement from patch A to patch $B$ by two alternative models:

$$
\begin{aligned}
\frac{d p}{d t}= & -m p N \exp \left[\lambda\left(W_{\mathrm{B}}-W_{\mathrm{A}}\right)\right] \\
& +m(1-p) N \exp \left[\lambda\left(W_{\mathrm{A}}-W_{\mathrm{B}}\right)\right], \\
\frac{d p}{d t}= & -m p N \exp \left(-\lambda W_{\mathrm{A}}\right) \\
& +m(1-p) N \exp \left(-\lambda W_{\mathrm{B}}\right),
\end{aligned}
$$

where $N$ is the total population size, $W_{j}$ is instantaneous per capita growth rate in habitat $j, p$ is the fraction of individuals in habitat $A, m$ is the basal per capita movement rate, and $\lambda$ is the sensitivity of movement to fitness. Equation (2a) implies that movement is based on a comparison of fitnesses in the two habitats, while equation (2b) implies that individuals leave one habitat at a more rapid rate when fitness is low but are unaffected by the current fitness in the other habitat. Ives (1992), de Roos et al. (2002), and Persson and de Roos (2003) use equation (2b) to describe the rate of leaving a patch. Equations (2a) and $(2 \mathrm{~b})$ both result in some movement to poorer-quality patches and thus do not meet CK's criteria for achieving an IFD in a single-species system.

Armsworth and Roughgarden $(2005 a, 2005 b)$ investigated a difference equation model of population dynamics that falls into the slow-model category. The per-individual movement rate from patch $i$ to $j$ in a two-patch system (which follows reproduction in the patch) was given by $m\left(w_{i}-w_{j}\right)$ when $w_{i}>w_{j}$ and by 0 when this inequality was reversed. Because of the discrete form of the model, their $w$ corresponds to $\exp (W)$ in equations (2). This movement rule does not allow movement to a poorerquality patch. A similar rule had been used by Bernstein et al. (1999) in a three-patch model using differential 
equations, but emigration from patch $i$ was given by $m\left(W^{*}-W_{i}\right)$ when $W_{i}<W^{*}\left(W^{*}\right.$ is the mean fitness across all patches) and was 0 when this inequality was reversed. These individuals then divide themselves evenly among those patches that have fitness greater than the mean. This rule also fits the CK criteria for achieving an IFD. Movement depends on the quality of the receiving patch indirectly because of its influence on the mean $W^{*}$. The property of no movement to lower-quality patches is also shared by the simulation studies of Schwinning and Rosenzweig (1990) and Mooij et al. (2002).

Both classes of model (rapid and slow) have the same variables: population sizes $N_{i}$ and proportional occupancy $p_{i}$. In some cases, the functions describing the dynamics of both variables are identical in spite of different assumptions about the timescale of habitat occupancy. This is illustrated by the "replicator dynamics" proposed by Taylor and Jonker (1978) and widely used in evolutionary game theory. Under this model, the dynamics of the proportion $p_{i}$ of type $i$ in the population are given by $d p_{i} / d t=p_{i}\left(W_{i}-W^{*}\right)$, where $W^{*}$ is again the mean fitness. CKG used replicator dynamics to illustrate the dynamics of approaching an IFD for a single species. This model associates fitness with each habitat (as in the slow models). Movement dynamics are not specified, and changes in occupancy come about because of the differential reproduction of different patches. As shown by CKG, this results in an equation for the change in occupancy of patch $A$ in a two-patch system, $d p / d t=p(1-p)\left(W_{1}-W_{2}\right)$, which is equivalent to $d p / d t=p(1-p)\left(d W^{*} / d p\right)$. This equivalency of fast and slow models does not occur in general. As noted by $\mathrm{CK}$, the replicator equations have the undesirable property of allowing fixation of the population in one habitat, because movement ceases when $p=1$ or 0 .

Within the set of movement models reviewed here, there are some models within each category that allow movement to lower-quality patches. Hugie and Grand (1998) reviewed empirical studies and found a small number of cases with no movement to lower-fitness habitats. However, these were characterized by widely separated habitats for which low rates were expected, and distinguishing between low and zero movement rates would have been difficult. In the case of diet choice within a habitat, the analogous prediction of zero consumption of the poorerquality prey was essentially never observed (Pyke 1984). Models considered here also differ in whether they assume some influence of other patches on movement out of a given patch. There is little evidence to distinguish between these alternatives, and most theoretical works mentioned above do not cite any empirical studies to justify one alternative over another.

Many previous models of habitat choice have no movement dynamics; they simply assume that such movement happens rapidly enough that habitat distributions reach a quasi equilibrium before population densities change significantly (Rosenzweig 1991; Abrams 1992; Křivan 1997; Krrivan and Sirot 2002; CKG; Egas et al. 2005). That equilibrium is generally assumed to be an IFD. However, the assumption of infinitely rapid distributional change that achieves a stable equilibrium is inconsistent with some models of movement. For instance, under replicator dynamics or other versions of equation (1) where the rate function $v$ becomes quite small for some values of $p$, behavioral change becomes very slow when almost all individuals are located in a subset of the habitats, even when it is advantageous to move. This is true, for example, of the models in Abrams (1999). Such prolonged lags might be plausible when extended exposure to one set of conditions reduces behavioral sampling (Abrams 1999) or when behavioral changes occur by imitation of the behavior of other individuals (Schlag 1997). In addition, it is not clear that it is always justified to assume that behavioral equilibrium is reached without significant population dynamical change (Abrams 2000). Moreover, there may be no stable behavioral equilibrium for fixed population sizes in some cases (see next section; Schwinning and Rosenzweig 1990; Abrams and Matsuda 1997), or the interaction of behavioral dynamics and population dynamics may significantly change the average values of both population sizes and behavioral traits (Abrams 2006a, 2006b).

There is experimental evidence showing that the analogous separation of timescales between evolutionary and population dynamical change does not occur (Yoshida et al. 2003). The question of the rate of change of the distribution of individuals among patches is particularly important in systems in which the environment fluctuates (e.g., Abrams 2000). Fluctuating environments also increase the importance of knowing the functional form of relationships between fitness differences and movement rates. There is often a nonlinear relationship between the magnitude of a difference between two states and the ability of an animal to perceive that difference (Getty and Krebs 1985), suggesting that the rate of behavioral change in response to a difference in those same two states will also be nonlinear, unlike the models of Armsworth and Roughgarden $(2005 a, 2005 b)$ and Bernstein et al. (1999), among others.

It is notable that early analyses of IFDs assumed that the resource population instantaneously reached an equilibrium for the current consumer population(s). In other words, the resource dynamics occurred on the same timescale as the behavioral redistribution of consumer individuals. The resulting "input matching rule" of Parker (1984) became the norm in many subsequent analyses of IFDs (e.g., Sih 1998). Analyses of food web interactions have found that predators may have slower dynamics than 
Table 1: Coexistence equilibria of the Cressman et al. (2004) figure 6 competition model (eqq. [3], [4])

\begin{tabular}{lrrrrl}
\hline Point & $N_{1}$ & \multicolumn{1}{c}{$N_{2}$} & \multicolumn{1}{c}{$p$} & \multicolumn{1}{c}{$q$} & $\begin{array}{c}\text { Per capita growth rates } W_{i j} \text { of } \\
\text { species } i \text { in habitat } j^{\mathrm{a}}\end{array}$ \\
\hline $1^{\mathrm{b}}$ & 11 & 11 & $10 / 11$ & $1 / 11$ & $W_{1 \mathrm{~A}}=W_{1 \mathrm{~B}}=W_{2 \mathrm{~B}}=W_{2 \mathrm{~A}}=0$ \\
2 & 20 & 10 & $19 / 20$ & 0 & $W_{1 \mathrm{~A}}=W_{1 \mathrm{~B}}=W_{2 \mathrm{~B}}=0 ; W_{2 \mathrm{~A}}>0$ \\
3 & 10 & 20 & 1 & $1 / 20$ & $W_{1 \mathrm{~A}}=W_{2 \mathrm{~B}}=W_{2 \mathrm{~A}}=0 ; W_{1 \mathrm{~B}}>0$ \\
4 & 1 & 12 & 0 & $1 / 6$ & $W_{1 \mathrm{~A}}=W_{2 \mathrm{~A}}=W_{1 \mathrm{~B}}=0 ; W_{2 \mathrm{~B}}>0$ \\
5 & 12 & 1 & $5 / 6$ & 1 & $W_{1 \mathrm{~A}}=W_{1 \mathrm{~B}}=W_{2 \mathrm{~A}}=0 ; W_{2 \mathrm{~B}}>0$ \\
6 & 19 & 19 & 1 & 0 & $W_{1 \mathrm{~A}}=W_{2 \mathrm{~B}}=0 ; W_{1 \mathrm{~B}}>0 ; W_{2 \mathrm{~A}}>0$ \\
7 & 2 & 2 & 0 & 1 & $W_{1 \mathrm{~B}}=W_{2 \mathrm{~A}}=0 ; W_{1 \mathrm{~A}}>0 ; W_{2 \mathrm{~B}}>0$ \\
8 & 10 & 1 & 1 & 1 & $W_{1 \mathrm{~A}}=W_{2 \mathrm{~A}}=0 ; W_{1 \mathrm{~B}}>0 ; W_{2 \mathrm{~B}}>0$ \\
9 & 1 & 10 & 0 & 0 & $W_{1 \mathrm{~B}}=W_{2 \mathrm{~B}}=0 ; W_{1 \mathrm{~A}}>0 ; W_{2 \mathrm{~A}}>0$ \\
\hline
\end{tabular}

a The per capita growth rates are given by equations (6).

b Unstable candidate ideal free distribution that would represent the equilibrium in the absence of movement.

their prey, but the maximum turnover rates generally differ by less than an order of magnitude (Yodzis and Innes 1992). This means that, if resources (prey) change on the same timescale as predator behavior, it is inconsistent to assume that the predator populations change on a much slower timescale than their own behavior.

\section{An Illustration of the Importance of Behavioral Dynamics for Population Dynamics}

CKG analyzed an example of the behavioral and population dynamics of two competitors to illustrate that the joint population dynamical equilibria in each of two isolated patches might not be stable when the patches were coupled by adaptive movement. However, behavioral and population dynamics were explored separately, so it was not clear how the combined behavioral-population system would change over time. This example is analyzed in more detail here to illustrate the importance of modeling the joint dynamics of populations and behaviors and also to illustrate that equally plausible movement rules can produce dramatically different population dynamics.

The system in question consists of two competing species that are each capable of growing in two different patches. The within-patch fitness is given by the LotkaVolterra competition equations, and so the populations change based on

$$
\begin{aligned}
\frac{d N_{1}}{d t}= & r_{1 \mathrm{~A}} p N_{1}\left(\frac{K_{1 \mathrm{~A}}-N_{1} p-\alpha_{12 \mathrm{~A}} N_{2} q}{K_{1 \mathrm{~A}}}\right)+r_{1 \mathrm{~B}}(1-p) N_{1} \\
& \times\left[\frac{K_{1 \mathrm{~B}}-N_{1}(1-p)-\alpha_{12 \mathrm{~B}} N_{2}(1-q)}{K_{1 \mathrm{~B}}}\right],
\end{aligned}
$$

$$
\begin{aligned}
\frac{d N_{2}}{d t}= & r_{2 \mathrm{~A}} q N_{2}\left(\frac{K_{2 \mathrm{~A}}-N_{2} q-\alpha_{21 \mathrm{~A}} N_{1} p}{K_{2 \mathrm{~A}}}\right)+r_{2 \mathrm{~B}} N_{2}(1-q) \\
& \times\left[\frac{K_{2 \mathrm{~B}}-N_{2}(1-q)-\alpha_{21 \mathrm{~B}} N_{1}(1-p)}{K_{2 \mathrm{~B}}}\right],
\end{aligned}
$$

where the species are labeled 1 and 2, habitats are A and $\mathrm{B}$, and $p$ and $q$ represent fractions of the populations in habitat A. In the example from CKG (their fig. 6), the parameters are $\alpha_{12 \mathrm{~A}}=9, \alpha_{12 \mathrm{~B}}=0.1, \alpha_{21 \mathrm{~A}}=0.1, \alpha_{21 \mathrm{~B}}=$ $9, \quad r_{1 \mathrm{~A}}=1, \quad r_{1 \mathrm{~B}}=0.1, \quad r_{2 \mathrm{~A}}=0.1, \quad r_{2 \mathrm{~B}}=1, \quad K_{1 \mathrm{~A}}=19$, $K_{1 \mathrm{~B}}=2, K_{2 \mathrm{~A}}=2$, and $K_{2 \mathrm{~B}}=19$. This set of parameters means that the two competitors are symmetric in their abilities in the two patches: competitor 1 has a higher carrying capacity but is more vulnerable to interspecific competition in patch A than in B. The reverse is true for competitor 2. CKG assumed that for each pair of population densities, the equilibrium distribution across habitats, given by $p$ and $q$, was achieved before any significant change in population densities. The single point where both competitors occupy both patches and have zero population growth $\left(N_{1}=N_{2}=11 ; p=10 / 11 ; q=1 / 11\right)$ was shown to be unstable; this pair of population sizes resulted in two alternative locally stable points where each species occupied a different habitat ( $p=1$ and $q=0$, or $q=$ 1 and $p=0$ ).

It is clear that neither of the species can be excluded globally, because each species can increase in either patch when it is rare and the other species is at its carrying capacity in both patches. To make some predictions about the dynamics of the system, we need to examine all of the potential coexistence equilibria to determine whether any of them can be stable, in terms of both population density and between-patch distribution. Table 1 lists the nine po- 
tential coexistence equilibria, including the candidate IFD point mentioned above, where both species are present globally in a system without movement. The eight additional points are characterized by having one or both species restricted to a single habitat. It is clear from the table that none of these equilibria is a two-species IFD because, in each case, the patch in which one species is missing has a higher payoff than the patch that is occupied by that species. We conclude that equations (3), augmented so that $p$ and $q$ change toward the patch currently having a higher fitness, have no stable equilibrium. This conclusion does not depend on the details of the movement rule, provided that the movement does not significantly perturb densities from the equilibria listed in the table. Because species cannot go extinct, as noted above, there must be fluctuations in their population sizes. However, the nature of the fluctuations then depends on the coupled dynamics of spatial distribution and population size. Furthermore, it is not clear that "nonideal" movement will produce the same population dynamical equilibria that are listed in table 1.

We illustrate the dynamics produced by several different models. We begin with a system in which the replicator equations (Taylor and Jonker 1978; CKG) describe the dynamics of the habitat distribution:

$$
\begin{aligned}
\frac{d p}{d t}=v_{1} p(1-p) & \left\{r_{1 \mathrm{~A}}\left(\frac{K_{\mathrm{1A}}-N_{1} p-\alpha_{12 \mathrm{~A}} N_{2} q}{K_{\mathrm{1A}}}\right)\right. \\
& \left.-r_{1 \mathrm{~B}}\left[\frac{K_{1 \mathrm{~B}}-N_{1}(1-p)-\alpha_{12 \mathrm{~B}} N_{2}(1-q)}{K_{1 \mathrm{~B}}}\right]\right\},
\end{aligned}
$$

$$
\begin{aligned}
\frac{d q}{d t}=v_{2} q(1-q) & \left\{r_{2 \mathrm{~A}}\left(\frac{K_{2 \mathrm{~A}}-N_{2} q-\alpha_{21 \mathrm{~A}} N_{1} p}{K_{2 \mathrm{~A}}}\right)\right. \\
& \left.-r_{2 \mathrm{~B}}\left[\frac{K_{2 \mathrm{~B}}-N_{2}(1-q)-\alpha_{21 \mathrm{~B}} N_{1}(1-p)}{K_{2 \mathrm{~B}}}\right]\right\},
\end{aligned}
$$

where $v_{i}$ is a scaling constant reflecting rates of behavioral change and $p$ and $q$ are the proportions of individuals in habitat A for species 1 and 2, respectively. When both $v_{i} \gg 1$, these equations represent a case in which behavioral change is much more rapid than population dynamical change. However, for fixed population densities, the absolute values of $v_{1}$ and $v_{2}$ simply reflect the temporal units and do not affect dynamics. Figure 1 shows the vector field in phase space produced by this pair of behavioral equations for the population densities at three of the final eight equilibria listed in table 1 plus the densities at equilibrium point $1\left(N_{1}=N_{2}=11\right)$. In all cases, the plots show that the values of $p$ and $q$ at the equilibrium (the center of the small circle in each panel) do not represent stable states for the behavioral dynamics. The point at $\left(N_{1}=N_{2}=11 ; p=10 / 11 ; q=1 / 11\right)$ is the only population dynamical equilibrium when there is movement and the only candidate IFD for both species, but as originally shown in CKG, the behavioral dynamics make this point unstable. CKG noted that this would result in complete habitat segregation ( $p=1$ and $q=0$, or $q=1$ and $p=0$ ). However, because these distributional equilibria are not population dynamical equilibria for $N_{1}=N_{2}=$ 11, it was not clear from CKG what long-term dynamics are expected in the system.

Although it was not possible to perform a global stability analysis of this four-dimensional system (eqq. [3], [4]), only three qualitatively different types of dynamics were observed in numerical integrations of parameter sets with equal rate parameters $v_{1}=v_{2}=v$. For small $v$ (less than approximately 2.6858), the equilibrium at $p=10 / 11$, $q=1 / 11$, and $N_{1}=N_{2}=11$ became locally (and apparently globally) stable. This is understandable based on the fact that the stable population dynamics dominate the unstable behavioral dynamics when the latter are slow enough. When $v$ was significantly larger than this threshold value, the system was characterized by large-amplitude cycles in which $p$ and $q$ were antiphase but the global densities of the two species fluctuated in phase. Figure 2 shows the dynamics that occur with a rate constant $(v=2.7)$ slightly above the stability threshold for initial conditions given in the figure legend. The exact trajectory depended on initial conditions, but all conditions eventually resulted in the cycles shown on the right-hand side of the figure. The amplitude of the cycles expands as the behavioral rate constants increase. The cycles can be understood from figure $1 D$, which shows the behavioral dynamics starting with both populations at the candidate IFD equilibrium point with $N_{i}=11$. The equilibrium at $p=1$ and $q=$ 0 means that both species occupy the habitat where they have a carrying capacity of 19 . When densities are close to this point, figure $1 A$ applies, and the species switch so that each is almost entirely in the habitat where its carrying capacity is 2. This leads to a population crash in both species. As they approach the carrying capacity, figure $1 C$ applies, and adaptive movement again provokes another switch of habitats.

A third outcome of numerical integrations was observed at large values of $v$. In this case, the dynamics were initially cyclic, but the frequencies of each species in a given habitat approached 0 or 1 so closely that the proportion of one species in one habitat was eventually rounded to 1 or 0 

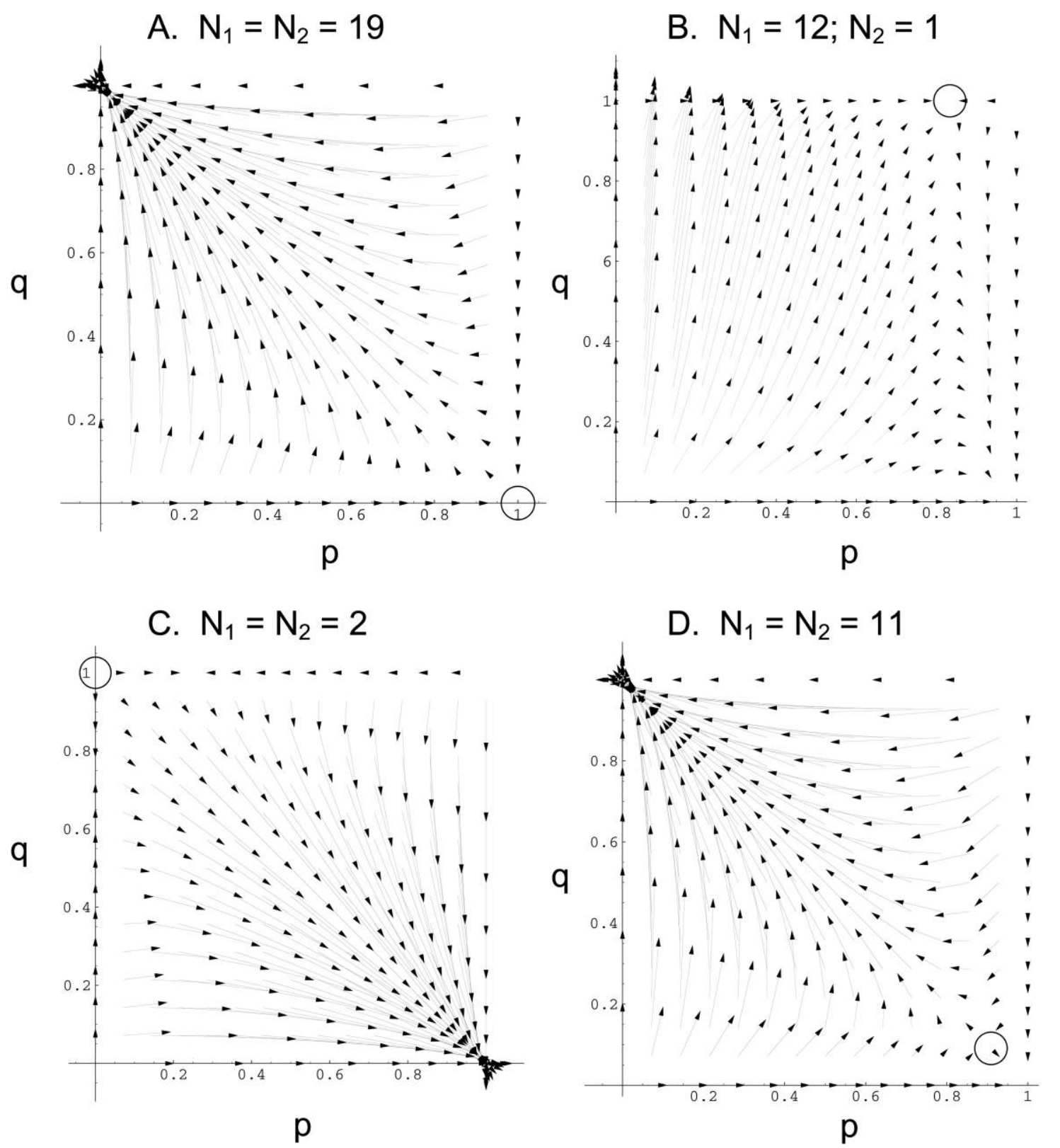

Figure 1: Vector fields showing the behavioral dynamics defined by the replicator equations for the cases from Cressman et al.'s (2004; CKG) figure 6 example for four different pairs of (constant) total population densities. Arrows give the direction and magnitude of the change in habitat distributions across a grid of potential distributions of the two species between two habitats, given the replicator dynamics of equations (4). In each case, the coexistence equilibrium being examined is characterized by a distribution of individuals that lies at the center of the small circle (see table 1). A, The point $p=1, q=0$ is not locally stable when $N_{1}=N_{2}=19$; the locally stable point for these population densities is $q=1, p=0$. However, this behavioral equilibrium is not a population dynamic equilibrium; both per capita growth rates are negative at this point. $B, N_{1}=$ 12 and $N_{2}=1$ has a population dynamical equilibrium point where most of the species-1 individuals are in patch 1 and all of the species-2 individuals are in patch $2(p=5 / 6 ; q=1)$, but the plot again shows that this is behaviorally unstable, with the system moving to $p=1, q=0$. $C, N_{1}=N_{2}=2$ has a population dynamical equilibrium at $p=0, q=1$, but the behavioral dynamics at this point are not at equilibrium; the plot shows that both species switch habitats completely to end up at $p=1, q=0$. Plot $D$ corresponds to the equilibrium studied in detail by CKG: $N_{1}=N_{2}=11, p=10 / 11, q=1 / 11$. However, this is again behaviorally unstable; the vector field shows that the system moves to either $p=1$, $q=0$ or $q=1, p=0$, depending on the initial deviation from the equilibrium. 

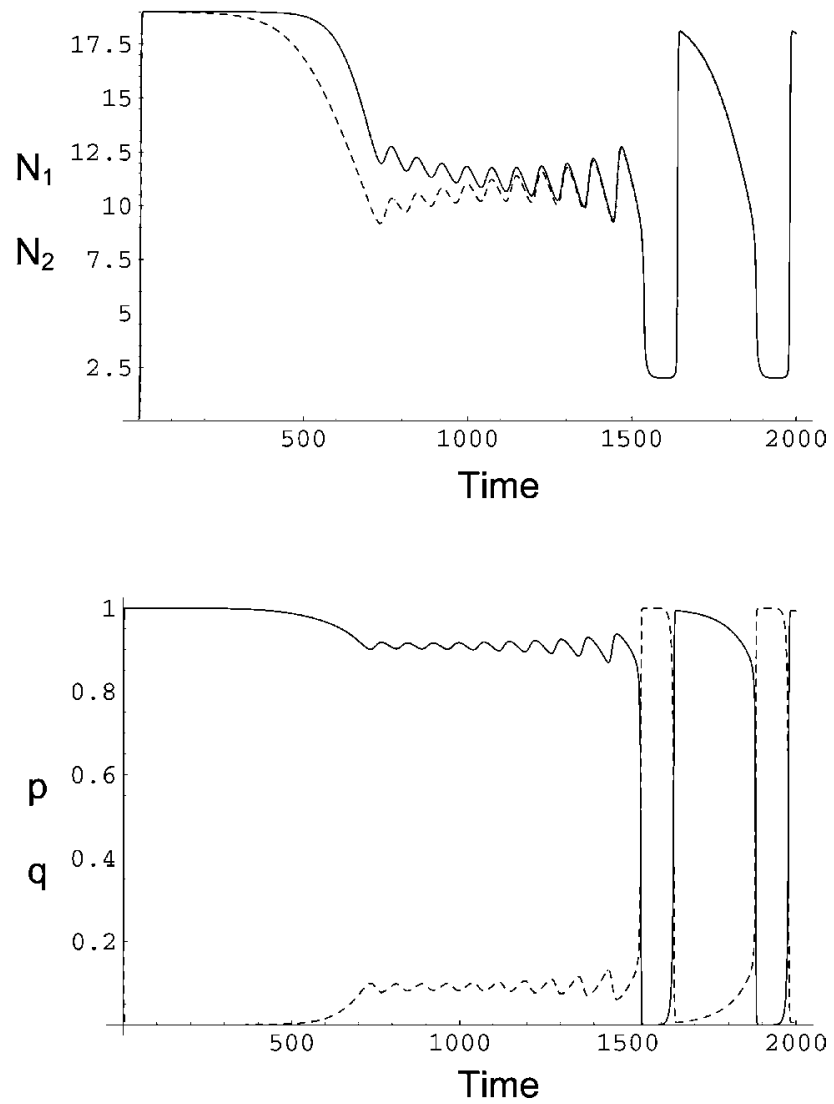

Figure 2: Dynamics of the full replicator dynamics model (eqq. [3], [4]) for the case of $v_{1}=v_{2}=2.7$, with parameters as in figure 1 (Cressman et al.'s [2004] fig. 6), and initial values $N_{2}=1, N_{1}=1, q=0.9$, and $p=0.9$. The ultimate dynamics are cycles, but the system first approaches the unstable equilibrium at $N_{1}=N_{2}=19, p=1, q=0$. It then approaches the second unstable equilibrium at $N_{1}=N_{2}=11$ with $p=$ $10 / 11$ and $q=1 / 11$, but the cycles around that point expand; the final dynamics shown appear to persist indefinitely. The top panel shows total population densities of the species (solid line, $N_{1}$; dashed line, $N_{2}$ ), and the bottom panel shows the proportions in habitat A (solid line, species 1; dashed line, species 2).

by the numerical integrator (Mathematica 5.2 [Wolfram 2005] command NDSolve with an AccuracyGoal of infinity; this occurred for $v=8$, given initial conditions close to the interior equilibrium point, and appears to occur for all initial conditions when $v=10$ ). Although a more accurate integrator may have yielded persistent cycles in this case, population densities become so low that the population of at least one species in one patch would become 0 in most finite populations. At that point, the cycles would stop, and the replicator dynamics would imply that the species that was absent from a patch could not recolonize it. The initial extinction in all of the numerical results occurred in the patch where a species had its greater car- rying capacity $(K=19)$. This was apparently because the lowest densities of a given species in a patch were observed when individuals moved out of their high- $K$ patch. Thus, the end result was a stable point with $p=5 / 6, q=1$, $N_{1}=12$, and $N_{2}=1$ or the "mirror image" point with $p=0, q=1 / 6, N_{1}=1$, and $N_{2}=12$. In other words, one species occurs alone in the habitat where it has lower growth parameters, and both species share the other habitat.

The replicator equations can be made somewhat more realistic by allowing behavioral mutation to occur. This may be accomplished by adding the term $\mu(1-p)-\mu p$ to equation (4a) and adding the same term, with $q$ substituted for $p$, to equation (4b). Random movement to the other habitat is one form of behavioral mutation that can be represented by such an expression. In this modified replicator model, three types of attractors were observed for the full four-equation system. The first involved cycles that were similar in form to those shown at the right-hand side of figure 2. Given initial populations close to the point with both species at equilibrium in two separate habitats $\left(p=10 / 11, q=1 / 11, N_{1}=N_{2}=11\right)$, cycles were observed for all values of $v$ larger than a threshold value that decreased with increasing values of the mutation parameter $\mu$. The minimum $v$ for cycles in the absence of mutation/random movement was approximately 2.6858; when $\mu=0.001$, that minimum $v$ was reduced to approximately 1.975 . However, a sufficiently large value of $\mu$ led to alternative stable points, as noted below. On the cycling attractor, larger values of $v$ made the cycles in $p$ and $q$ more closely approach a square waveform, as shown by the difference between figure $3 A$ and figure $3 B$. The period of the cycles also decreases with increasing $v$; for $v=500$ in figure $3 B$, the period is approximately an order of magnitude less than the period for $v=4$ in figure $3 \mathrm{~A}$. More rapid behavior changes the relative duration of the two phases of this population cycle in such a way that each species spends a larger proportion of its time being dominant in the habitat where it has a lower carrying capacity; this proportion increases from 0.2633 in figure $3 A$ to 0.7358 in figure $3 B$. Thus, the mean population densities of both species decline significantly as their rate of behavioral change increases, from 13.600 in figure $3 \mathrm{~A}$ to 6.535 in figure $3 B$.

The second type of outcome for replicator equations with added random movement occurred when initial densities were close to the candidate IFD point but behavioral change was slower; here, there was a stable equilibrium with equal total populations of the two species but with each species predominant in the habitat where it had the larger carrying capacity. The total density of each species was only slightly under 11 (the candidate IFD density) in models with random movement, when the rate parameter 

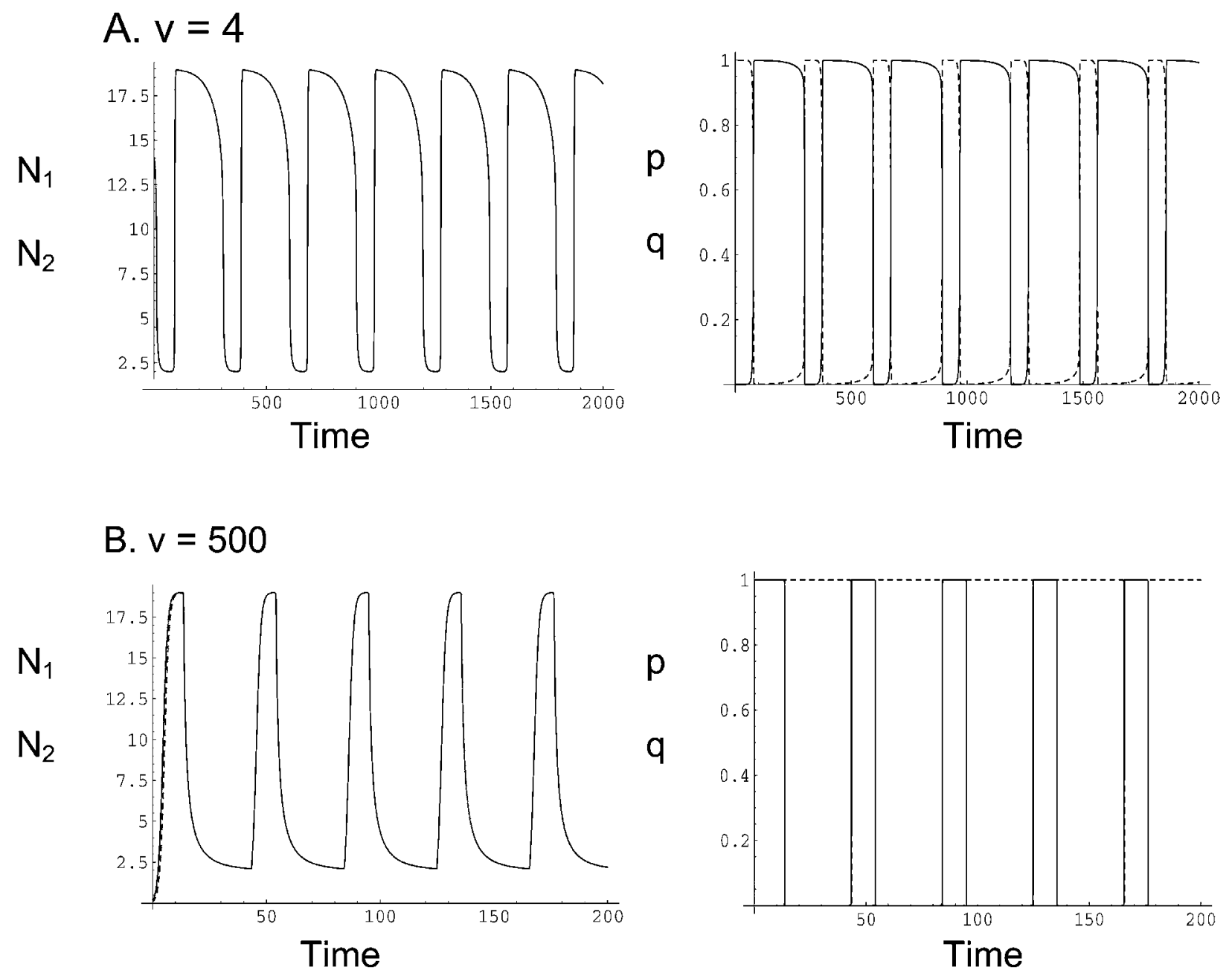

Figure 3: Dynamics of the full replicator dynamics model (eqq. [3], [4]), augmented with a behavioral mutation rate of $\mu=10^{-6}$ for the cases of $v_{1}=v_{2}=4$ and $v_{1}=v_{2}=500$, with parameters as in figure 1 (Cressman et al.'s [2004] fig. 6). Note that the time axis spans 2,000 time units for $A$ but 200 time units for $B$. The left-side plots show total population densities of each species (solid line, $N_{1}$; dashed line, $N_{2}$ ), and the right-side plots show the proportions in habitat A (solid line, species 1; dashed line, species 2). Because the fluctuations in total population size for the two species are synchronous, the two lines are superimposed.

$\mu$ was very close to 0 , but it decreased rapidly as $\mu$ increased. For example, $\mu=0.001$ produced equilibrium densities of 6.851 when $v=1$.

The final type of outcome was a pair of locally stable equilibria in which one species was much more abundant than the other and both were found mainly in the habitat where the abundant species had a higher $K$. A larger value of $\mu$ was required to produce this third outcome when the rates of adaptive habitat selection, $v$, were larger. Often the less abundant species was excluded entirely. If $v=3$ and $\mu=0.01$, one of the equilibrium points is $p=0.8662$, $q=0.9737, N_{1}=17.666$, and $N_{2}=0.4158$; the other (mirror image) point is $p=0.0263, q=0.1338, N_{1}=$
0.4158, and $N_{2}=17.666$. Each outcome reflects the source-sink dynamics that occur when one species reaches a high enough density in the habitat where it has a high $K$; migrants to the other habitat, combined with the large competitive effect in that other habitat, greatly reduce or exclude the second species globally. (Similar outcomes in a random-movement model were observed by Amarasekare and Nisbet [2001] and by Abrams and Wilson [2004].) This particular set of rate constants also had a cyclic attractor similar to that illustrated in figure 3 , which was reached when initial conditions were close to the interior equilibrium at $p=10 / 11$ and $q=1 / 11$.

Figure 4 shows the dynamics of the same model of 
competition after assuming that movement between habitats is described by equation (2a), for two choices for the movement constants $m$ and $\lambda$. The distributional dynamics are given by the following two equations:

$$
\begin{aligned}
\frac{d p}{d t}= & p(1-p)\left(W_{1 \mathrm{~A}}-W_{1 \mathrm{~B}}\right)+m_{1}(1-p) \\
& \times \exp \left[\lambda_{1}\left(W_{1 \mathrm{~A}}-W_{1 \mathrm{~B}}\right)\right]-m_{1} p \exp \left[\lambda_{1}\left(W_{1 \mathrm{~B}}-W_{1 \mathrm{~A}}\right)\right]
\end{aligned}
$$

$$
\begin{aligned}
\frac{d q}{d t}= & q(1-q)\left(W_{2 \mathrm{~A}}-W_{2 \mathrm{~B}}\right)+m_{2}(1-q) \\
& \times \exp \left[\lambda_{2}\left(W_{2 \mathrm{~A}}-W_{2 \mathrm{~B}}\right)\right]-m_{2} q \exp \left[\lambda_{2}\left(W_{2 \mathrm{~B}}-W_{2 \mathrm{~A}}\right)\right]
\end{aligned}
$$

where the fitnesses are given by the per capita growth rates from the Lotka-Volterra equations:

$$
\begin{aligned}
& W_{1 \mathrm{~A}}=r_{1 \mathrm{~A}}\left(\frac{K_{1 \mathrm{~A}}-N_{1} p-\alpha_{12 \mathrm{~A}} N_{2} q}{K_{1 \mathrm{~A}}}\right), \\
& W_{1 \mathrm{~B}}=r_{1 \mathrm{~B}}\left[\frac{K_{1 \mathrm{~B}}-N_{1}(1-p)-\alpha_{12 \mathrm{~B}} N_{2}(1-q)}{K_{1 \mathrm{~B}}}\right], \\
& W_{2 \mathrm{~A}}=r_{2 \mathrm{~A}}\left(\frac{K_{2 \mathrm{~A}}-N_{2} q-\alpha_{21 \mathrm{~A}} N_{1} p}{K_{2 \mathrm{~A}}}\right), \\
& W_{2 \mathrm{~B}}=r_{2 \mathrm{~B}}\left[\frac{K_{2 \mathrm{~B}}-N_{2}(1-q)-\alpha_{21 \mathrm{~B}} N_{1}(1-p)}{K_{2 \mathrm{~B}}}\right] .
\end{aligned}
$$

The dynamics of the total population size are still given by equations (3). We assume here that the two species share the same value of $m$ and the same $\lambda$. The baseline movement rate $m$ is somewhat analogous to the randommovement parameter in the extended replicator equation; it specifies the per capita rate of movement when both habitats confer equal fitness. However, $m$ also affects the speed of adaptive change. If $m$ is relatively small but the sensitivity to fitness difference $\lambda$ is large, the system does not exhibit cycles, unlike the corresponding case for equations (3) and (4) (i.e., the four-dimensional model with replicator dynamics for behavior). Instead, there are three alternative locally stable equilibrium points: (1) the point that approximates the candidate IFD point; (2) two points at which one of the two species is totally absent or very rare while the other exhibits "undermatching" (Hugie and Grand 1998), in that it is slightly below its carrying capacity in its high- $K$ habitat and slightly above its $K$ in its low- $K$ habitat. These three points correspond qualitatively to the stable equilibrium points for the model with replicator equations augmented by random movement. An example of the approach to one of the near-exclusion equilibria is shown in figure $4 A$. Cycling similar in form to that shown in figure 3 occurs for relatively high fitness sensitivity and relatively high baseline movement, as shown in figure $4 B$ ( $m=0.1, \lambda=5)$. However, the cycles have a much shorter period than cycles produced by replicator dynamics with small mutation/random movement rates, even for the very high values of $v$ shown in figure $3 B$. The cycling outcome when equation ( $2 \mathrm{a}$ ) defines behavior also differs from cycles under the replicator equations in having lower cycle amplitudes. It is worth noting that raising the fitness difference in the exponential terms of equation (2a) to the power of 3 (making behavioral dynamics relatively less sensitive to small differences in fitness and more sensitive to large differences) eliminates cycles entirely, given the parameters used in figure $4 B$.

This same example was explored using several additional slow-behavior models. Using equation (2b) for the movement model produced a range of dynamics similar to those shown in figure 4, although there are quantitative differences. The cycles produced in this case do not result in as large a concentration of species in their high- $K$ habitats, and so the mean densities are much lower (and cycle periods are much shorter). Another slow model can be produced by changing equation ( $2 a$ ) so that it satisfies the requirements sufficient for the stability of a singlespecies IFD presented in CK. In this case, the movement rate from habitat $\mathrm{A}$ to habitat $\mathrm{B}$ is $m p\left\{N \exp \left[\lambda\left(W_{\mathrm{B}}-\right.\right.\right.$ $\left.\left.\left.W_{\mathrm{A}}\right)\right]-1\right\}$ if $W_{\mathrm{B}}>W_{\mathrm{A}}$ and 0 if $W_{\mathrm{B}} \leq W_{\mathrm{A}}$. The ArmsworthRoughgarden model, mentioned above (Armsworth and Roughgarden 2005a, 2005b), is a simpler model that also satisfies the CK criteria for attaining a single-species IFD. Simulations were carried out using both of these models, and they produced results with substantial quantitative differences from those produced by equation (2a). For example, low movement rates in the Armsworth-Roughgarden model resulted in the candidate IFD point being locally (and apparently globally) stable. The dynamics at higher movement rates were more like those of the replicator equations than those of equation (2a). This seems to be the result of the lack of movement to lower-fitness patches. Although many more models of movement and models of interaction could be explored, the numerical analysis of several movement rules for this example of two-species competition is sufficient to show that the type of behavioral dynamics can have a large effect on population dynamics. 

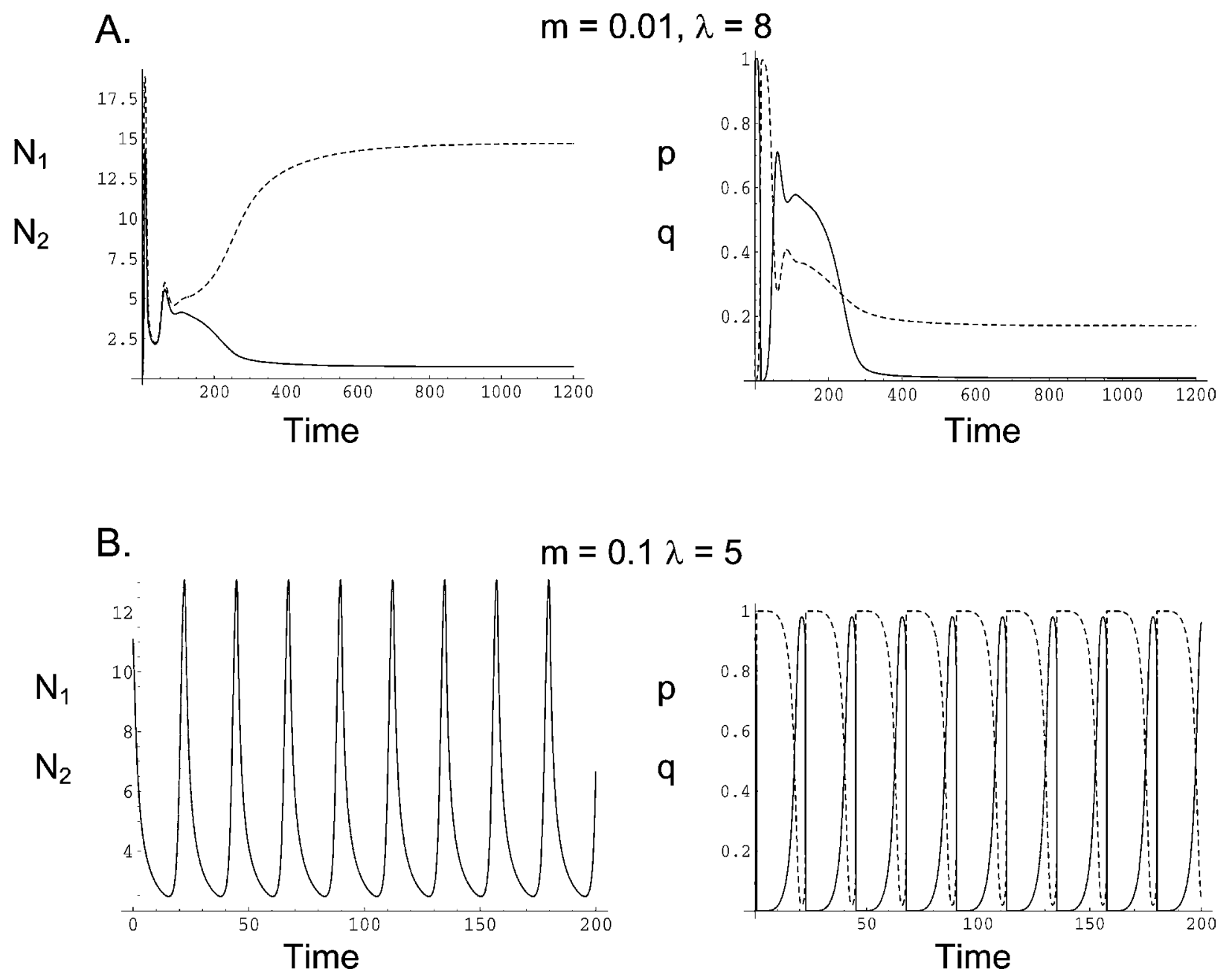

Figure 4: Dynamics of the competition model with movement based on equation (2a) (equivalently, eqq. [5], [6]) for two pairs of movement parameters $m$ and $\lambda$. The plots show the proportions of both species in habitat A (right) and the total population densities across both patches (left; populations are synchronous in $B$ ). As in figure 3, species 1 is described by the solid line and species 2 is described by the dashed line. Initial densities are close to the candidate ideal free distribution point: $N_{1 \mathrm{~A}}=10, N_{1 \mathrm{~B}}=1, N_{2 \mathrm{~A}}=1$, and $N_{2 \mathrm{~B}}=10.01$. In each case shown, the dynamics are only one of three alternative attractors that exist for each system.

\section{How Representative Are the Results from This Example of Two-Species Competition?}

The type of cycling observed in this system is likely to be relatively rare in two-patch Lotka-Volterra competitive systems. Cycles here depend on the very large difference in competition coefficients between the two patches and on the fact that each species has a much greater effect on its competitor in the habitat where its own intrinsic growth rate and carrying capacity are low. The instability of the candidate IFD that drives the cycles occurs because the movement of a small number of species- $j$ individuals into the patch where $i$ has a high density greatly reduces the fitness of $i$ in that patch, prompting movement of $i$ out of that patch and thus further immigration of species $j$. It is straightforward to show that cycles cannot occur in the Lotka-Volterra competition model (under any of the types of trait dynamics discussed here) when each competition coefficient has the same value in both patches (Křivan and Sirot 2002; CKG). In a more typical case of two-species Lotka-Volterra competition, it is likely that two habitats will differ in some parameters but not have the parameter values that can destabilize an internal equilibrium. For these cases, a system with some random component to its movement will likely cause departures from equal fitness across habitats, given nonzero movement to poorer patches. A large random component has 
the potential to greatly alter the outcome of the interaction (Amarasekare and Nisbet 2001; Abrams and Wilson 2004). However, most metapopulation models based on two-species Lotka-Volterra competition will not exhibit the cycles shown here. The conditions for stability of IFDs in systems having three or more competitors are still largely unknown.

There is a considerable literature arguing that the consumer-resource models are more appropriate than the Lotka-Volterra equations for most cases of resource competition between two or more competitors (Schoener 1986). For such models, there is considerable evidence that distributions having equal fitness may be stable or unstable, depending on the dynamics of behavior. Consumer-resource interactions are normally characterized by saturating consumer functional responses (Jeschke et al. 2002, 2004). As pointed out by Matsuda and Abrams (1994) and Abrams and Matsuda (1997), a type-2 consumer functional response introduces instability in the resource species' behavioral dynamics, and this often drives fluctuations in both populations (Schwinning and Rosenzweig 1990; Abrams 2007). When some or all of the resources are biological species, the system may have a candidate IFD where both species are distributed equally across the two patches when these have similar environmental parameters. However, the saturating consumer response makes it advantageous for their prey to aggregate, leading to two alternative stable IFDs where the prey are mostly or entirely in one of the two patches. Abrams (2007) shows that, even for fixed population densities, the equilibrium at which both predator and prey are present and equally fit in both patches is unstable for a very wide range of parameters. Because food webs contain many predator-prey links, it is likely that the nature of behavioral dynamics will be crucial in predicting habitat distributions of many species. Because competitors are often simply consumers that share living resources, this same mechanism is likely to operate for many sets of competitors.

\section{Concluding Remarks}

One of the more general questions related to this work on habitat selection is whether adaptive behavior must be taken into account in studying the population dynamics of interacting species (Bolker et al. 2003). CKG used a comparison of systems with no movement and systems with adaptive movement to argue for the importance (or unimportance) of behavior in determining habitat distributions. This interpretation is valid if the state without adaptive behavior is taken to be one with no movement. However, it could equally well be argued that the appropriate comparison is between systems with random or adaptive movement. It has long been known that random movement of individuals in metapopulations generally changes population sizes in patches, compared to the same system without movement (Levin 1974; Holt 1985). Random movement can also greatly alter interactions between competing species in a patchy habitat because of sourcesink phenomena (Amarasekare and Nisbet 2001; Abrams and Wilson 2004). Introducing even a small amount of random migration (i.e., using eqq. [2] with $\lambda=0$ and $m \ll 1)$ creates new population dynamical equilibria in the two-patch Lotka-Volterra example analyzed here. These altered equilibria represent one of the reasons why it is important to know something about the behavioral dynamics of movement, which may include a large or a small component of random movement. There are likely to be very few systems of interacting species in heterogeneous environments in which introducing adaptive movement does not alter the population sizes produced by random movement. This conclusion remains true regardless of whether an IFD is achieved by some or all species in the case of adaptive movement.

An important reason for modeling behavioral and population dynamics together is the fact that almost all populations fluctuate significantly in population size (Pimm 1991), and the population dynamics of any species will depend in part on how rapidly it can adapt behaviorally to changes in the spatial pattern of abundance of predators, competitors, and resources. There will always be some lag between changes in populations and/or growth parameters and achieving the new behavioral attractor. Previous work on adaptive movement of one of several interacting species (Abrams 1999, 2000; Abrams and Matsuda 2004) clearly shows that the details of behavioral dynamics (both functional form and rate constants) can have a major effect on such basic quantities as mean population sizes in systems with fluctuating populations. That message is reinforced by the example analyzed here. What is new here is the fact that the local population fluctuations can themselves be driven by the dynamics of adaptive movement.

Fretwell and Lucas (1970) clearly hoped that their conceptual framework of the "ideal free distribution" could provide a general explanation for habitat distributions. It is now clear that many species are not characterized by IFDs (Kennedy and Gray 1993), but the reasons for the deviations and the magnitudes of those deviations remain subjects of contention. We hope that this work will motivate behavioral biologists to consider interspecific interactions as one of the potential factors that can prevent attainment of IFDs in natural systems. This will require more work to determine the appropriate model of the behavioral dynamics of movement. 


\section{Acknowledgments}

P.A.A. and R.C. thank the Natural Sciences and Engi $\rightarrow 225$. neering Research Council of Canada for financial support. V.K. was supported by the Grant Agency of the Czech Academy of Sciences (A100070601). P.A.A. thanks W. G. Wilson for comments on an early draft that grew into this comment. structure of the environment. Journal of Animal Ecology 60:205225. decisions, the ideal free distribution, and predator-prey dynamics. American Naturalist 153:267-281.

Bolker, B. M., M. Holyoak, V. Křivan, L. Rowe, and O. Schmitz. 2003. Connecting theoretical and empirical studies of traitmediated interactions. Ecology 84:1101-1114.

$\rightarrow$ Briggs, C. J., and M. F. Hoopes. 2004. Stabilizing effects in spatial parasitoid-host and predator-prey models: a review. Theoretical Population Biology 65:299-315.

\section{Literature Cited}

$\rightarrow$ Cressman, R., and V. Křivan. 2006. Migration dynamics for the ideal free distribution. American Naturalist 168:384-397 (CK).

$\rightarrow$ Abrams, P. A. 1992. Predators that benefit prey and prey that harn predators: unusual effects of interacting foraging adaptations. American Naturalist 140:573-600.

$\rightarrow-1999$. The adaptive dynamics of consumer choice. Americar $\rightarrow$ Naturalist 153:83-97.

$\rightarrow-2000$. The impact of habitat selection on the heterogeneity of resources in varying environments. Ecology 81:2902-2913.

- 2003. Can adaptive evolution or behavior lead to diversification of traits determining a trade-off between foraging gain and predation risk? Evolutionary Ecology Research 5:653-670.

$\rightarrow-2006 a$. The effects of switching behavior on the evolutionary diversification of generalist consumers. American Naturalist 168 . 645-659.

$\rightarrow-2006 b$. The prerequisites for and likelihood of generalistspecialist coexistence. American Naturalist 167:329-342.

- 2007. Habitat choice in predator-prey systems: spatial instability due to interacting adaptive movements. American Nat uralist (forthcoming).

$\rightarrow$ Abrams, P. A., and H. Matsuda. 1993. Effects of adaptive predatory and anti-predator behavior in a two prey-one predator system Evolutionary Ecology 7:312-326.

$\rightarrow$. 1997. Prey evolution as a cause of predator-prey cycles. Evolution 51:1740-1748.

$\rightarrow-$ 2004. Consequences of behavioral dynamics for the population dynamics of predator-prey systems with switching. Population Ecology 46:13-25.

$\rightarrow$ Abrams, P. A., and W. G. Wilson. 2004. Coexistence in metacommunities due to spatial variation in resource growth rates: does $R^{*}$ predict the outcome of competition? Ecology Letters 7:929940.

Alonzo, S. H. 2002. State-dependent habitat selection games between predators and prey: the importance of behavioural interaction, and expected lifetime reproductive success. Evolutionary Ecology 4:759-778.

$\rightarrow$ Amarasekare, P., and R. Nisbet. 2001. Spatial heterogeneity, source sink dynamics, and the local coexistence of competing species. American Naturalist 158:572-584.

$\rightarrow$ Armsworth, P. R., and J. E. Roughgarden. 2005a. Disturbance induces the contrasting evolution of reinforcement and dispersiveness in directed and random movers. Evolution 59:2083-2096.

$\rightarrow-2005 b$. The impact of directed versus random movement on population dynamics and biodiversity patterns. American Naturalist 165:449-465.

$\rightarrow$ Bernstein, C., A. Kacelnik, and J. R. Krebs. 1988. Individual decisions and the distribution of predators in a patchy environment. Journal of Animal Ecology 57:1007-1026.

$\rightarrow-1991$. Individual decisions and the distribution of predators in a patchy environment. II. The influence of travel costs and
Cressman, R., V. Křivan, and J. Garay. 2004. Ideal free distributions, evolutionary games, and population dynamics in multiple-species environments. American Naturalist 164:473-489 (CKG).

de Roos, A. M., K. Leonardsson, L. Persson, and G. G. Mittelbach. 2002. Ontogenetic niche shifts and flexible behavior in size-structured populations. Ecological Monographs 72:271-292.

$\rightarrow$ Egas, M., M. W. Sabelis, and U. Dieckmann. 2005. Evolution of specialization and ecological character displacement of herbivores along a gradient of plant quality. Evolution 59:507-520.

Fretwell, S. D. 1972. Populations in a seasonal environment. Princeton University Press, Princeton, NJ.

$\rightarrow$ Fretwell, S. D., and H. L. Lucas Jr. 1970. On territorial behavior and other factors influencing habitat distribution in birds. Acta Biotheoretica 19:16-36. models of movement by Serengeti grazers. Ecology 85:2429-2435.

Fryxell, J. M., J. F. Wilmshurst, A. R. E. Sinclair, D. T. Haydon, R. D. Holt, and P. A. Abrams. 2005. Landscape scale, heterogeneity, and the viability of Serengeti grazers. Ecology Letters 8:328-335.

Getty, T., and J. R. Krebs. 1985. Lagging partial preferences for cryptic Naturalist 125:39-60.

Grimm, V., and S. F. Railsback. 2005. Individual-based modeling and ecology. Princeton University Press, Princeton, NJ.

$\rightarrow$ Holt, R. D. 1985. Population dynamics in two-patch environments: some anomalous consequences of optimal habitat distribution. Theoretical Population Biology 28:181-208.

Holyoak, M., M. A. Leibold, and R. D. Holt, eds. 2005. Metacommunities: spatial dynamics and ecological communities. University of Chicago Press, Chicago.

Hugie, D. M., and T. Grand. 1998. Movement between patches, unequal competitors, and the ideal free distribution. Evolutionary Ecology 12:1-19.

$\rightarrow$ Ives, A. R. 1992. Continuous-time models of host-parasitoid interactions. American Naturalist 140:1-29.

$\rightarrow$ Jeschke, J. M., M. Kopp, and R. Tollrian. 2002. Predator functional responses: discriminating between handling and digesting prey. Ecological Monographs 72:95-112.

$\rightarrow-$. 2004. Consumer-food systems: why type I functional responses are exclusive to filter feeders. Biological Reviews 79:337349 .

$\rightarrow$ Kennedy, M., and R. D. Gray. 1993. Can ecological theory predict the distribution of foraging animals? a critical analysis of experiments on the ideal free distribution. Oikos 68:158-166.

$\rightarrow$ Křivan, V. 1997. Dynamic ideal free distribution: effects of optimal patch choice on predator-prey dynamics. American Naturalist 149: 164-178.
Fryxell, J. M., J. F. Wilmshurst, and A. R. E. Sinclair. 2004. Predictive prey: a signal-detection analysis of great tit foraging. American 
$\rightarrow$ Křivan, V., and E. Sirot. 2002. Habitat selection by two competin species in a two-habitat environment. American Naturalist 160: 214-234.

$\rightarrow$ Levin, S. A. 1974. Dispersion and population interactions. American Naturalist 108:207-228.

$\rightarrow$ Matsuda, H., and P. A. Abrams. 1994. Timid consumers: selfextinction due to adaptive change in foraging and anti-predator effort. Theoretical Population Biology 45:76-91.

$\rightarrow$ Mooij, W. M., R. E. Bennetts, W. M. Kitchens, and D. L. DeAngelis. 2002. Exploring the effect of drought extent and interval on the Florida snail kite: interplay between spatial and temporal scales. Ecological Modelling 149:25-39.

Parker, G. A. 1984. Evolutionarily stable strategies. Pages 30-61 in J. R. Krebs and N. B. Davies, eds. Behavioural ecology: an evolutionary approach. 2nd ed. Sinauer, Sunderland, MA.

$\rightarrow$ Persson, L., and A. M. de Roos. 2003. Adaptive habitat use in sizestructured populations: linking individual behavior to population processes. Ecology 84:1129-1139.

Pimm, S. L. 1991. The balance of nature. University of Chicago Press, Chicago.

$\rightarrow$ Pyke, G. H. 1984. Optimal foraging theory: a critical review. Annual Review of Ecology and Systematics 15:523-575.

Rosenzweig, M. L. 1991. Habitat selection and population interactions: the search for mechanism. American Naturalist 137(suppl.): S5-S28.
Schlag, K. 1997. Why imitate, and if so, how? Journal of Economic Theory 78:130-156.

$\rightarrow$ Schoener, T. W. 1986. Mechanistic approaches to community ecology: a new reductionism? American Zoologist 26:81-106.

$\rightarrow$ Schwinning, S., and M. L. Rosenzweig. 1990. Periodic oscillations in an ideal-free predator-prey distribution. Oikos 59:85-91.

Sih, A. 1998. Game theory and predator-prey response races. Pages 221-238 in L. A. Dugatkin and H. K. Reeve, eds. Game theory and animal behavior. Oxford University Press, Oxford.

Taylor, P., and L. B. Jonker. 1978. Evolutionarily stable strategies and game dynamics. Mathematical Biosciences 40:145-156.

$\rightarrow$ van Baalen, M., and M. Sabelis. 1993. Coevolution of patch selection strategies of predators and prey and the consequences for ecological stability. American Naturalist 142:646-670.

$\rightarrow-1999$. Nonequilibrium population dynamics of "ideal and free" prey and predators. American Naturalist 154:69-88.

Wolfram, S. 2005. Mathematica 5.2. Wolfram Research, Champaign, IL.

$\rightarrow$ Yodzis, P., and S. Innes. 1992. Body size and consumer-resource dynamics. American Naturalist 139:1151-1175.

$\rightarrow$ Yoshida, T., L. E. Jones, S. P. Ellner, G. F. Fussman, and N. G. Hairston. 2003. Rapid evolution drives ecological dynamics in a predatorprey system. Nature 424:303-306.

Associate Editor: Peter D. Taylor Editor: Donald L. DeAngelis 